\title{
Disentangling component spectra of $\kappa$ Sco, a spectroscopic binary with a pulsating primary ${ }^{\star}$
}

\section{Improved physical elements and analysis of periodic rapid variations of scalar quantities}

\author{
P. Harmanec ${ }^{1,2}$, K. Uytterhoeven ${ }^{3}$, and C. Aerts ${ }^{3}$ \\ 1 Astronomical Institute of the Charles University, V Holešovičkách 2, 18000 Praha 8, Czech Republic \\ e-mail: hec@sunstel.asu.cas.cz \\ 2 Astronomical Institute, Academy of Sciences, 25165 Ondřejov, Czech Republic \\ 3 Instituut voor Sterrenkunde, Katholieke Universiteit Leuven, Celestijnenlaan 200 B, 3001 Leuven, Belgium
}

Received 12 February 2004 / Accepted 11 April 2004

\begin{abstract}
A new reduction and a new analysis of a rich series of high- $S / N$ spectra of the bright star $\kappa$ Sco is carried out to test the technique of spectral disentangling in the case when one of the components is a non-radial oscillator. We improve the orbital elements of the system and find the basic physical properties of the binary to be $T_{\text {eff }}=24500 \mathrm{~K}$, masses of 10.7-11.9 $M_{\odot}$ and 9.6-10.7 $M_{\odot}$ and the primary radius and rotational period of 7-8 $R_{\odot}$ and 3.56-3.68, respectively. This also implies $\log g=3.70-3.78$ [cgs]. KOREL disentangling is applied and we find that it worked properly and was not misled by the complex line-profile variability of the pulsating primary. Moreover, the pulsational frequencies are detected via the period search in the line intensities and radial-velocity residuals of the primary derived by KOREL and in the time series of residual spectra in the rest frame of the primary after KOREL disentangling. This constitutes a much better starting point to interpret the complex pixel-by-pixel variations of the disentangled profiles of the primary in terms of stellar oscillations and/or additional rotational modulation than the original spectra before disentangling.
\end{abstract}

Key words. stars: binaries: spectroscopic - stars: oscillations - line: profiles - stars: individual: $\kappa$ Sco

\section{Introduction}

The B1.5III star $\kappa$ Sco (HD 160578, HR 6580, HIP 86670) is a very bright $\left(V=2^{\mathrm{m}} \cdot 4\right) \beta$ Cep light variable, known from the study of Shobbrook \& Lomb (1972). Later, Lomb \& Shobbrook (1975) showed that the light variations over several years could be reconciled with a stable period of 0.1998303 and a sinusoidal light curve. From its amplitude variation with a period of 7.3 they concluded that the light changes are multiperiodic, the other short period being 0.205430 . They also showed that the first period could also be detected in radialvelocity ( $R V$ hereafter) changes during one night of spectroscopic observations.

Large $R V$ variations, exceeding the range of $\beta$ Cep oscillations, were reported by Moore et al. (1916) and again by Buscombe \& Morris (1960). Referring to their own and Buscombe \& Morris observations, Lomb \& Shobbrook (1975) concluded that the star is a spectroscopic binary. Comparing line-profiles of $\kappa$ Sco with synthetic ones, Heasley et al. (1982)

\footnotetext{
* Based on spectral observations obtained at ESO with the CAT/CES, La Silla, Chile.
}

remarked that the observed profiles are peculiar and that the object might be a double-lined spectroscopic binary. It was only De Mey (1997) who found that the $R V \mathrm{~s}$ vary with a period of $195^{\mathrm{d}} .8$ and derived the first orbital elements.

A detailed study of the orbital as well as rapid spectral variations, based on more than 400 high-resolution electronic spectra of Si III 4552 and $4567 \AA$ lines secured between 1988 and 1997, was published by Uytterhoeven et al. (2001) Paper A hereafter. The principal results of that study are:

1. $\kappa$ Sco is a double-lined spectroscopic binary with a 195.65 period, moving in an eccentric orbit $(e=0.488$, $\left.\omega=92^{\circ} 7\right)$ with a semiamplitude of the primary $K_{1}=$ $48.0 \mathrm{~km} \mathrm{~s}^{-1}$.

2. A weak and broad line from the secondary was suspected moving across the line profiles at different orbital phases. From various estimates (without being able to measure the $R V$ curve of the secondary), they arrived at the following probable basic physical properties of the binary: masses of 17 and $12 M_{\odot}$, radii of 6.8 and $5.8 R_{\odot}(\log g=4.0$ for both stars) and effective temperatures of 23400 and $18800 \mathrm{~K}$. 
3. Analysis of the first three moments of the line profiles showed that they are modulated by both frequencies found by Lomb \& Shobbrook (1975) and their various harmonics and aliases, and the presence of additional oscillation frequencies was suspected.

4. Using the moment method and line-profile fitting they concluded that the primary component of $\kappa$ Sco pulsates with a main prograde sectoral mode of degree 1 or 2 and an additional tesseral mode, most probably with wavenumbers $l=5$ and $m=1$.

Cuypers et al. (2004) recently analyzed two short datasets obtained with the star camera of satellite WIRE and found three other significant periods (besides the two already known ones): $3.5600,0.4445$ and 0.1756 . The nature of the first new period, which is too long to be identified with a typical $\beta$ Cephei pulsation, is still unknown. Below, we provide some arguments that it can probably be identified as the rotational period of the primary.

The initial motivation of this study was to use the technique of line-profile disentangling (Hadrava 1995, 1997) to derive not only the $R V$ curves of both binary components and improved orbital elements but also to analyze the residual spectra from KOREL disentangling in the rest frame of the primary to arrive at a better description of the line-profile changes. This study is meant to be a test case of how powerful the disentangling technique can be in application to complex line-profile variability that occurs in many early-type binaries. We also hoped to clarify the question of whether the secondary also is a line-profile variable. However, after the first attempts at KOREL disentangling, we encountered problems and we finally decided to reinvestigate the original spectrograms. Since their continua have often a complicated wavy character and are strongly tilted in the outer parts of the spectrum, we carried out a new rectification interactively using the SPEFO program (see Horn et al. 1996; Škoda 1996). Each newly rectified spectrum was immediately compared to a sample spectrum to ensure the most uniform rectification. Only then we did apply KOREL and repeated analyses of the rapid periodic variations of integrated quantities, such as the radial velocity and line intensity. These results are reported in this paper. In a subsequent study, we will analyse the complex short-period line-profile variability of the primary after the KOREL disentangling in full detail and we will identify the oscillation modes (Uytterhoeven et al., in preparation).

\section{Observations and data reduction}

The observational material used in this study consists of a homogeneous set of 699 high-resolution CCD spectra obtained at ESO, La Silla, with the Coudé Auxiliary Telescope (CAT) and Coudé Echelle Spectrometer (CES). They were used by Uytterhoeven et al. (2001). They are well distributed in the orbital phase. All spectra contain the Si III triplet near $4560 \AA$. However, the Si III $4575 \AA$ line is much noisier and on a number of spectra it is also affected by bad pixels of the CCD. For that reason, our analyses were restricted to Si III 4552 and Si III 4567 Å lines only.
Table 1. Journal of available $R V \mathrm{~s}$ of $\kappa \mathrm{Sco}$.

\begin{tabular}{ccrc}
\hline \hline $\begin{array}{c}\text { Observatory } \\
\text { and source }\end{array}$ & $\begin{array}{c}\text { Time interval } \\
(\text { HJD-2 400 000) }\end{array}$ & $\begin{array}{r}\text { No. of } \\
\text { spectra }\end{array}$ & $\begin{array}{c}\text { Dispersion } \\
\left(\AA \mathrm{mm}^{-1}\right)\end{array}$ \\
\hline 1 & $17787.53-21100.49$ & 9 & $10.3-37.4$ \\
2 & $34944.01-35269.09$ & 4 & 36 \\
3 & $40765.03-40765.24$ & 12 & $3.9-6.8$ \\
4 & $47297.76-50742.47$ & 752 & $R=57000$ \\
\hline
\end{tabular}

Column "Observatory and source":

1: Lick southern station in Chile: Cerro San Cristóbal 0.929-m reflector, 1-, 2- and 3-prism spectrographs (Campbell \& Moore 1928); 2: Mt. Stromlo 0.762-m reflector, Cassegrain 3-prism spg. (Buscombe \& Morris 1960); 3: Mt. Stromlo 1.88-m reflector, Coudé grating spg. (Lomb \& Shobbrook 1975); 4: ESO Coudé auxiliary telescope, CES CCD spg. (Paper A).

\section{Improving the value of the orbital period}

As mentioned above, the studies of three earlier sets of $R V \mathrm{~s}$ of $\kappa$ Sco are published, and fortunately all sets are available. Combined, they cover the whole range of $R V$ variation over the $195^{\mathrm{d}} 6$ period, although on the rising branch only. We therefore decided to use them for the determination of a more accurate value of the orbital period which could then be kept fixed in all subsequent analyses.

The journal of available $R V \mathrm{~s}$ is given in Table 1 . For $R V \mathrm{~s}$ published by Campbell \& Moore (1928) we used mean $R V \mathrm{~s}$ where the same plate was measured several times. Note also the misprint in Table IV of Lomb \& Shobbrook (1975): The correct time of the mid-exposure of the second $R V$ is HJD 2440765.0375 , not HJD 2440765.0875 , as we have verified with the help of their Fig. 6.

To take widely different dispersions and resolutions of different spectrograms into account, we assigned weights to individual $R V \mathrm{~s}$ which are directly proportional to spectral resolution.

The spectral resolution is basically given by the FWHM (full width at half maximum) of the projected slit of the spectrograph. It can also be expressed as

$R=\frac{\lambda}{n \cdot \mathrm{d} \lambda}=\frac{\lambda}{n \cdot D \cdot s}$

where $\lambda$ is the central wavelength of the spectrum in question in $\AA, D$ is its linear dispersion in $\AA \mathrm{mm}^{-1}, \mathrm{~d} \lambda$ is the wavelength difference of the projected spectrum between the two neighbouring detection elements (grain size or pixel spacing) in $\AA, s$ is the "pixel" spacing in mm and $n$ is the number of $\mathrm{d} \lambda$ per FWHM of the projected slit width, typically $2-3$. The usual values of $s$ for photographic emulsions range between 0.020 and $0.025 \mathrm{~mm}$.

Considering this, we adopt a value of $s=0.020 \mathrm{~mm}$ and $n=2$ for the photographic spectra and estimate their spectral resolution from formula (1), adopting $4340 \AA$ for their approximate central wavelength. For electronic spectra, we adopt their mean resolution of 57000 (see Paper A).

We assigned weights to all $R V \mathrm{~s}$ according to the formula

$w=\frac{R}{20000}$. 
Table 2. Exploratory orbital solutions: All epochs are given in HJD-2 400 000, the rms errors of the solutions are rms errors of 1 observation of unit weight and the systemic velocities are identified by the observatory codes from Table 1.

\begin{tabular}{|c|c|c|c|c|c|}
\hline $\begin{array}{l}\text { Solution: } \\
\text { element }\end{array}$ & $\begin{array}{l}\text { 1: Paper A } \\
\text { published }\end{array}$ & $\begin{array}{l}\text { 2: Paper A } \\
\text { FOTEL }\end{array}$ & 3: older $R V \mathrm{~s}$ & $\begin{array}{l}\text { 4: all data } \\
\text { 1-d normals }\end{array}$ & $\begin{array}{l}\text { 5: all } 699 \text { spectra } \\
\text { KOREL }\end{array}$ \\
\hline$P$ & 195.65 & $195.712 \pm 0.041$ & $195.555 \pm 0.024$ & $195.4579 \pm 0.0068$ & 195.4579 fixed \\
\hline$T_{\text {periastr. }}$ & $49983.9 \pm 0.3$ & $49984.01 \pm 0.43$ & $29258.8 \pm 3.7$ & $49984.17 \pm 0.41$ & 49984.18 \\
\hline$T_{\max . R V}$ & not given & 49962.16 & 29245.63 & 49965.00 & 49964.03 \\
\hline$T_{\min . R V}$ & not given & 50003.17 & 29289.99 & 50002.16 & 50002.00 \\
\hline$e$ & $0.488 \pm 0.005$ & $0.4755 \pm 0.0071$ & 0.4755 fixed & $0.5100 \pm 0080$ & 0.503 \\
\hline$\omega$ & $92.7 \pm 0.5$ & $93: 6 \pm 0.89$ & $66^{\circ} .5 \pm 9.0$ & $91.7 \pm 1.1$ & $93: 3$ \\
\hline$K_{1}\left(\mathrm{~km} \mathrm{~s}^{-1}\right)$ & $48.0 \pm 0.3$ & $48.51 \pm 0.42$ & $49.8 \pm 1.8$ & $46.87 \pm 0.46$ & 54.94 \\
\hline$K_{2}\left(\mathrm{~km} \mathrm{~s}^{-1}\right)$ & - & - & - & - & 60.90 \\
\hline$\gamma_{1}\left(\mathrm{~km} \mathrm{~s}^{-1}\right)$ & - & - & $+6.0 \pm 1.5$ & $+4.1 \pm 2.0$ & - \\
\hline$\gamma_{2+3}\left(\mathrm{~km} \mathrm{~s}^{-1}\right)$ & - & - & $-3.4 \pm 1.4$ & $-1.0 \pm 3.0$ & - \\
\hline$\gamma_{4}\left(\mathrm{~km} \mathrm{~s}^{-1}\right)$ & $+0.2 \pm 0.1$ & $+0.47 \pm 0.19$ & - & $+0.08 \pm 0.28$ & - \\
\hline $\mathrm{rms}\left(\mathrm{km} \mathrm{s}^{-1}\right)$ & not given & 2.97 & 2.53 & 2.13 & $2.66 / 8.58$ \\
\hline
\end{tabular}

One should also take into account that the $R V$ accuracy increases for a higher $S / N$ ratio but with the limited data at hand we believe this is counterbalanced by a higher number of lines measured in the photographic spectra.

To derive various trial and then final orbital solutions, we used the latest version of the computer program FOTEL, developed by Hadrava (1990) ${ }^{1}$. FOTEL estimates the errors of the elements on the basis of a covariance matrix and allows determination of individual systemic velocities as elements of the solution.

First we derived the orbital solution for all $752 R V$ s already used in Paper A (53 Reticon and 699 CCD spectra). In Table 2 we compare this solution to the one given in Paper A, obtained with the Léhmann-Filhés method. This gives some idea of what the differences between different codes for $R V$ solution can be. Keeping the eccentricity from the FOTEL solution fixed, we then derived a solution for the older $R V$ s from photographic spectra. This solution is also given in Table 2. Note that we derived a joint systemic velocity for both Mt. Stromlo instruments. The practical reason for this is that the spectra from the $1.88-\mathrm{m}$ reflector cover only one night of observations and would not be of any value for constraining the period if allowed to have their own zero point. We verified, however, that our approach is fully justified. Running a trial solution in which allowance for individual systemic velocities was made, we found both Mt. Stromlo instruments to have nearly identical zero points, the difference being $0.6 \mathrm{~km} \mathrm{~s}^{-1}$, well below the errors of both values.

Taken at face value, the comparison of the solutions for the older and more recent data seems to indicate apsidal advance and also an increase of the orbital period. However, considering the small number of older observations, their heterogeneity and also the fact that rapid line-profile variations affect the

\footnotetext{
1 The program is made freely available to interested users by the author. Its description, Fortran source code and user's guide can be obtained via http://www. asu.cas.cz/ ^had/had.html
}

measured $R V \mathrm{~s}$, we adopt a conservative approach here and assume that the orbit has remained constant over the time interval covered by the data.

The $R V$ maxima and $R V$ minima are obviously the best defined time instants in the observed $R V$ curve of $\kappa \mathrm{Sco}$. It is easy to verify that within the errors of the epochs for the older and more recent data, separated for 106 orbital cycles, the time difference between $R V$ maxima calls for values of the orbital period between $195^{\mathrm{d}} .40$ and $195^{\mathrm{d}} .48$ and the difference between the minima for values between 195.37 and 195.47 . (Note that one-year aliases for 105 and 107 orbital cycles would be 193.6 and 197.3 which are values safely excluded by the electronic spectra.) This simple exercise indicates that both data sets can be combined to derive a more accurate value of the orbital period. It is clear, however, that to obtain a reasonable balance between the older and much more numerous recent data, one should not use the $R V \mathrm{~s}$ as they are. Only for the purpose of determination of a new value of the orbital period we therefore replaced the original data set of $R V \mathrm{~s}$ by the nightly normals, keeping however their original weights assigned to individual data. This solution is given as the fourth one in Table 2 and the corresponding $R V$ curve is shown in Fig. 1. It is clearly seen that the older and more recent data can indeed be well reconciled with a constant period.

\section{KOREL disentangling}

To disentangle the spectra and to derive orbital elements, we used the most recent version of the program KOREL - see Hadrava $(1995)^{2}$ - to which we also implemented calculation of weighted rms errors of $R V \mathrm{~s}$ derived via cross-correlation of

\footnotetext{
2 The program is made freely available to interested users by the author. Its more detailed description, Fortran source code and user's guide can be obtained via

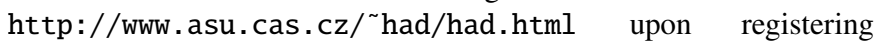
yourself at had@sunstel.asu.cas.cz
} 


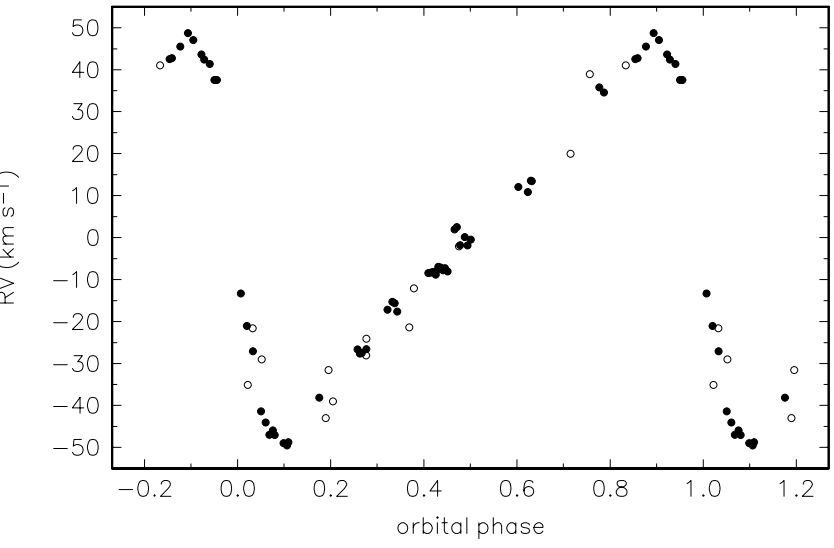

Fig. 1. The radial-velocity curve of $\kappa$ Sco for $1-\mathrm{d} R V$ normals. The $R V \mathrm{~s}$ from older photographic spectra are shown by empty circles.

the final disentangled spectrum with the individual stellar spectra. The current version of KOREL does not allow calculation of the errors of orbital elements. Note that the basic principle on which the KOREL disentangling is carried out is that the line profiles of the binary components can vary in intensity but not in their shape. This condition is clearly violated in the case of line-profile variables. However - as it was already pointed out by Harmanec et al. (1997) - as long as the time scale of line-profile variations is much shorter than the orbital period and the variations are not strictly phase-locked with the orbital motion, they can simply be considered a high-frequency noise and disentangling of the mean line profiles from rich enough series of spectra should still be possible. All this is true in the particular case of $\kappa$ Sco. This is why we believe that our application of KOREL is justified.

There is another complication in this particular case, however, because the amplitude of the line-profile variations is comparable to the strength of the line profile of the secondary. This leads to a situation that the sum of squares of residuals of the KOREL solution varies very slowly with the binary mass ratio, passing through a number of local minima of comparable depths.

We ran a number of trial solutions mapping the parameter space. It turned out that the mass ratio must be higher than the one estimated in Paper A. The most satisfactory fits were obtained for solutions leading to mass ratios between about 0.9 and 1.1 and we finally arrived at a solution which is given (for comparison) as the fifth one in Table 2. The corresponding $R V$ curves are shown in Fig. 2. Some larger residuals from the $R V$ curve of the secondary are not surprising. KOREL derives the velocities via cross-correlation of individual line profiles with the disentangled ones. In cases when a subfeature with a strength comparable to that of the secondary line profile appears near the position of the secondary line, the code is fooled and returns an incorrect $R V$ for the secondary.

Nevertheless, to illustrate the good overall performance of KOREL, we show the comparison of the disentangled profiles with the observed ones for two opposite elongations of the binary in Figs. 3 and 4.
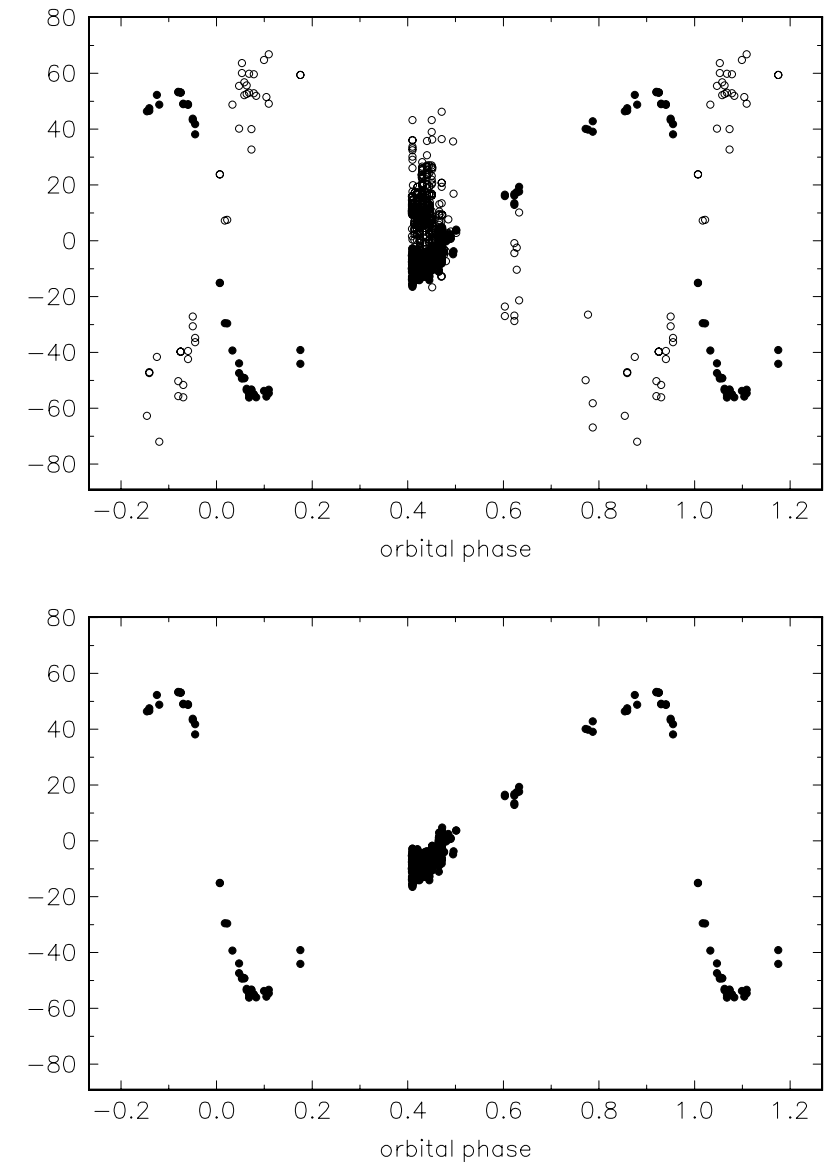

Fig. 2. The radial-velocity curve of $\kappa$ Sco for all 699 individual spectra (circles) based on KOREL disentangling. The upper panel shows the $R V$ curves of both binary components (secondary by empty symbols) while the bottom panel shows the same $R V$ curve for the primary only for clarity. The data are plotted with respect to ephemeris from the KOREL solution $T_{\text {periastr. }}=$ HJD $2449984.18+195^{\mathrm{d}} 4579 \times E$.

\section{Estimates of basic physical elements}

There are several circumstances that do not allow us to derive any of the basic physical elements of the binary and its components accurately: we only have at our disposal spectra from a very limited spectral region, there are no interferometric observations and the inclination of the orbit is unknown. We also do not know the relative luminosities of the components. Finally, the mass ratio we derived is still preliminary. Under these circumstances we can only set certain limits on the binary properties.

Johnson et al. (1966) give the following mean $U B V$ values: $V=2^{\mathrm{m}} \cdot 41, B-V=-0^{\mathrm{m}} \cdot 21$ and $U-B=-0^{\mathrm{m}} .88$. The Hipparcos photometry transformed to the Johnson $V$ via Harmanec's (1998) transformation formula gives $V=2^{\mathrm{m}} .386$, in excellent agreement with the Geneva photometry mean value $V=2^{\mathrm{m}} .382$. Adopting these latter values and Johnson et al.'s colour indices, one obtains the following dereddened values: $V_{0}=2^{\mathrm{m}} .284$, $B-V_{0}=-0^{\mathrm{m}} .242$ and $U-B_{0}=-0^{\mathrm{m}} .903$. These are normal values for a B1.5III star (cf., e.g., Golay 1974) and Popper's (1980) calibration implies $T_{\text {eff }}=24500 \mathrm{~K}$. 

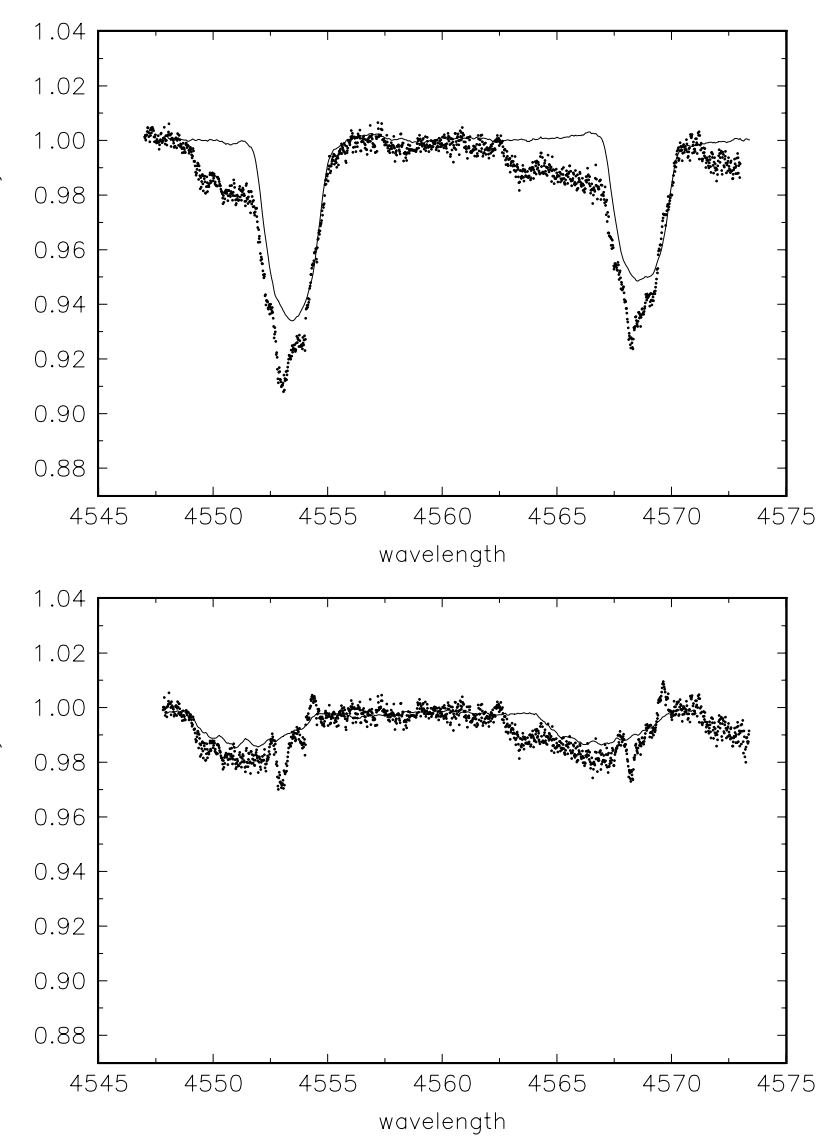

Fig. 3. The spectrum obtained at one elongation on HJD 2450742.4691 is first compared with the disentangled Si III line profile of the primary shifted in $R V$ for $+52.8 \mathrm{~km} \mathrm{~s}^{-1}$ (upper panel). The residual spectrum after subtraction of the disentangled profile of the primary is compared with the disentangled profile of the secondary shifted for $-58.5 \mathrm{~km} \mathrm{~s}^{-1}$ (bottom panel).

Combining the definition of the effective temperature $T_{\text {eff }}$

$M_{\text {bol }}=42.368-5 \log \left(R / R_{\odot}\right)-10 \log T_{\text {eff }}$

with the distance modulus and bolometric correction $B C$ interpolated from Code et al. (1976)

$V_{0}-M_{\mathrm{V}}=-5 \log \pi-5$,

one gets a working equation

$\log \left(R / R_{\odot}\right)=7.474-2 \log T_{\text {eff }}-0.2 \cdot\left(B C-V+A_{\mathrm{V}}\right)-\log \pi$.

As pointed out by Harmanec (2000), the fortunate circumstance is that the function $2 \log T_{\text {eff }}+0.2 B C$ varies quite slowly for hot stars. This implies that even for an approximate estimate of $T_{\text {eff }}$ one obtains a rather accurate value of the radius provided the visual magnitude $V$, reddening $A_{\mathrm{V}}$ and parallax $\pi$ are known with good accuracy ${ }^{3}$.

3 The numerical values used in these formulæ and in orbital solutions derived by FOTEL are based on the solar bolometric magnitude of $+4^{\mathrm{m}} .75$, accurate value of solar mass of $(1.988435 \pm 0.000027) \times$ $10^{30} \mathrm{~kg}$ and the gravitational constant $G=(6.674215 \pm 0.000092) \times$ $10^{-11} \mathrm{~m}^{3} \mathrm{~kg}^{-1} \mathrm{~s}^{-2}$ derived by Gundlach \& Merkowitz (2000) and on
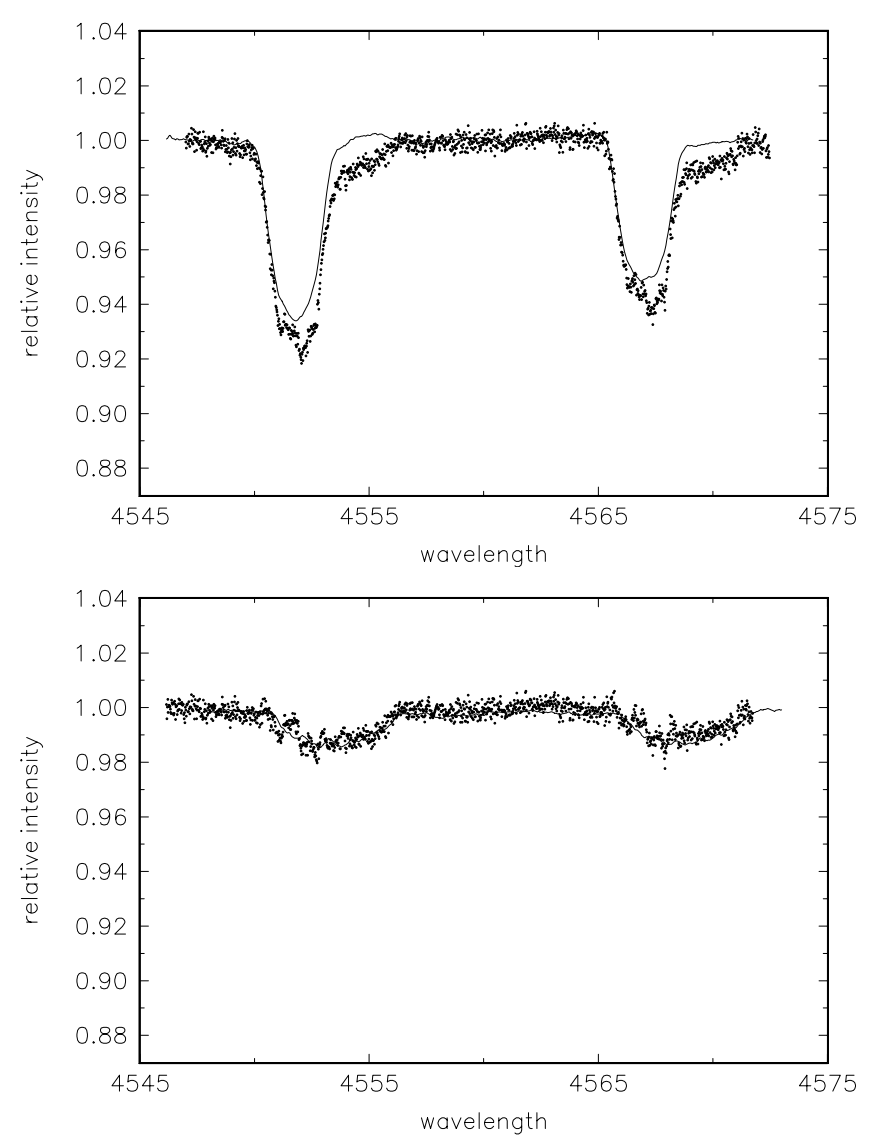

Fig. 4. The spectrum obtained at the other elongation on HJD 2450194.9224 is first compared with the disentangled Si III line profile of the primary shifted in $R V$ for $-56.1 \mathrm{~km} \mathrm{~s}^{-1}$ (upper panel). The residual spectrum after subtraction of the disentangled profile of the primary is compared with the disentangled profile of the secondary shifted for $+62.0 \mathrm{~km} \mathrm{~s}^{-1}$ (bottom panel).

Adopting the Hipparcos parallax of $p=0 . .^{\prime} 00703 \pm$ 0 '00073 after Perryman et al. (1997) and the above values, one arrives at a stellar radius of $7.6 R_{\odot}$, the range given by the parallax error being $6.9-8.5 R_{\odot}$. For comparison, using $T_{\text {eff }}=23400 \mathrm{~K}$ (to have some idea about possible colour effects of a fainter secondary on the observed $U B V$ magnitudes) would lead to a very similar radius: $8.0(7.3-8.9) R_{\odot}$. According to Harmanec's (1988) tabulation, a normal mass range for these effective temperatures would be (8.8-9.7) $M_{\odot}$. The problem of these estimates is that they represent only an upper limit of the radius of the $\kappa$ Sco primary since we do not know the contribution of the secondary to the luminosity of the system. However, one can also estimate the lower limit of the radius of the primary, assuming that both stars are equally bright in the $V$ passband, i.e. assuming the $V$ magnitude of the primary to be for $2.5 \log 2=0.752$ fainter than the dereddened $V$ magnitude of the system. For $T_{\text {eff }}=24500 \mathrm{~K}$ and the observed parallax this leads to the radius of the primary of 5.4 (4.9-6.0) $R_{\odot}$, which is still larger than the normal main-sequence radius of a star with such effective temperature which, according to

the solar radius of $6.95835 \times 10^{8} \mathrm{~m}$ derived by Harmanec from a fit of numerous measurements of the solar angular radius available in the astronomical literature (to be published). 
Harmanec's (1988) tabulation, is $4.6 R_{\odot}$. Notably, the range of possible values of the primary radius is not too wide, especially if one considers that the primary is probably the brighter of the two.

The orbital solution from KOREL leads to $M_{1} \sin ^{3} i=$ $10.69 M_{\odot}$ and $M_{2} \sin ^{3} i=9.64 M_{\odot}$. The inclination of the oscillation symmetry axis of $40^{\circ}$ estimated in Paper A from lineprofile modelling seems unacceptably low since it would imply masses over $35 M_{\odot}$.

In Fig. 6 we compare the probable radius and $T_{\text {eff }}$ of the primary with Schaller's et al. (1992) evolutionary models. One can see that the most probable range of the mass of the primary is between about 10 and $12 M_{\odot}$ which in turn implies that the orbital inclination should not be smaller than about $75^{\circ}$. Available Hipparcos photometry does not cover phases near the upper conjunction of the binary. We, therefore, cannot exclude the presence of at least partial eclipses and $90^{\circ}$ remains a practical upper limit on the inclination. This leaves us with a range $10.7-11.9 M_{\odot}$ for the primary and 9.6-10.7 $M_{\odot}$ for the secondary. The difference in masses is certainly sufficient for the assumption that (due to a strong dependence of the mainsequence lifetime on the mass) the secondary is still a luminosity class $\mathrm{V}$ object with a radius significantly smaller than the primary.

A comparison of disentangled line profiles with the synthetic spectra indicates projected rotational velocities of the binary components $v_{1} \sin i=100 \mathrm{~km} \mathrm{~s}^{-1}$ and $v_{2} \sin i=170 \mathrm{~km} \mathrm{~s}^{-1}$. Assuming that the equatorial plane of the primary and the orbital plane coincide, and that the primary star contributes 90 per cent of the total light in the $V$ passband, one has a range of $i=75-90^{\circ}$ also for the inclination of the rotational axis of the primary. For $T_{\text {eff }}=24500 \mathrm{~K}$ and the Hipparcos parallax, formula (5) gives $R_{1}=7.28 R_{\odot}$. The corresponding range of the the rotational period of the primary is then between 3.56 and 3.68 . This brackets the value of 3.56 detected in the WIRE photometry by Cuypers et al. (2004). This is why we believe that the observed 3.56 period can probably be identified with the rotational period of the $\kappa$ Sco primary. Note also that a spin-orbit synchronization at periastron would require a rotational period of the primary of some 56 days. Clearly, the system is far from the spin-orbit synchronization at periastron.

In passing we note that $\kappa$ Sco is obviously a good target for interferometric observations. In the optimal case, not only the orbital inclination but also the relative luminosities of the components could be derived and this in turn would allow a much more accurate determination of the component masses and radii. On the other hand, with its relatively short and accurately enough known orbital period, it could become a good calibration target for future interferometers.

\section{Rapid radial-velocity, line-intensity and light variations}

As is obvious from Figs. 3 and 4, we can immediately answer one of the questions we posed. The line-profile variations occur only over the wavelength range corresponding to the instantaneous position of the primary line profile. We conclude that

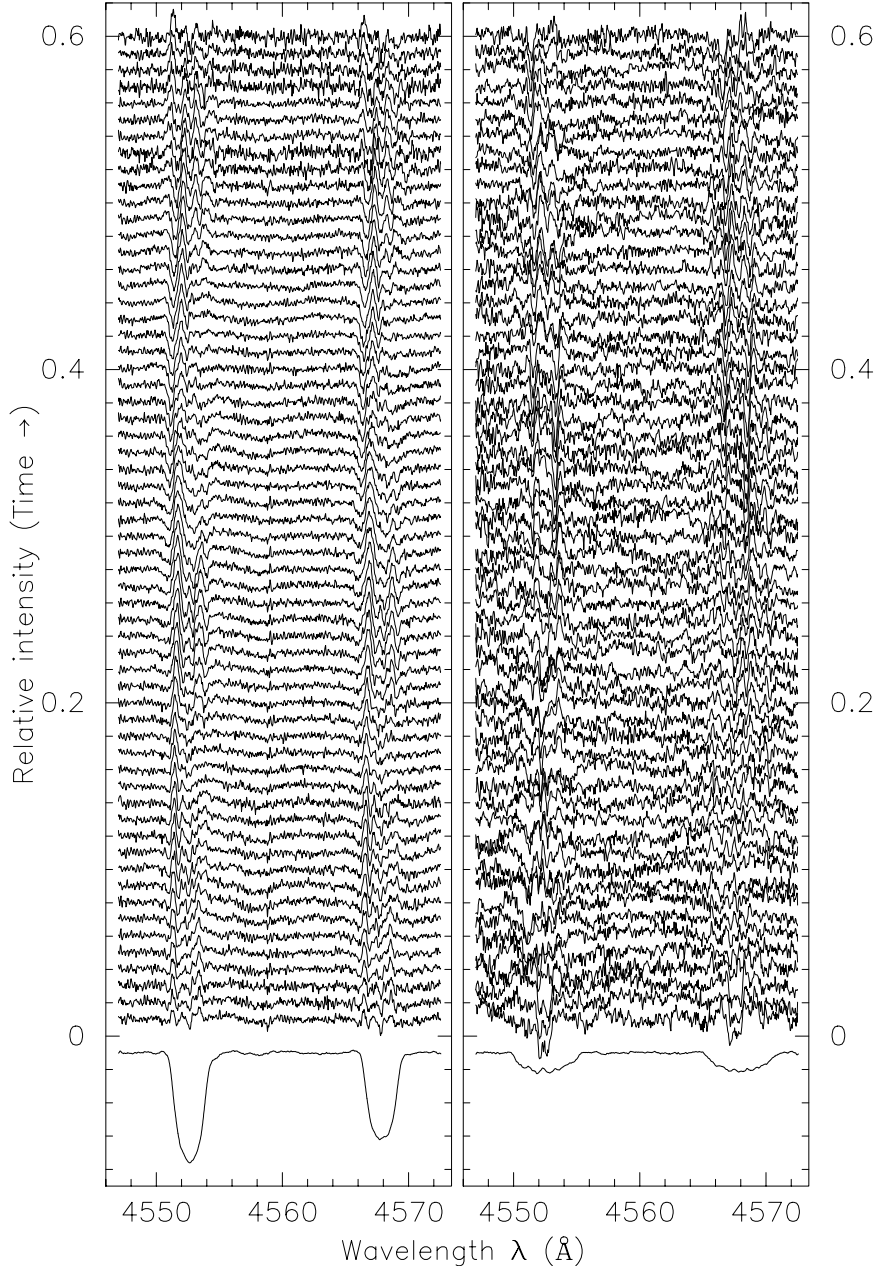

Fig. 5. Residual line profiles from the spectra taken during the night of 20 July 1997 (HJD 2450650 ) after KOREL disentangling. The left (right) panel shows the profiles in the rest frame of the primary (secondary). The disentangled Si III profile of the primary (secondary) is shown at the bottom of the panel. Time runs from the bottom to the top.

- at least on the noise level of our spectra - only the primary component is the line-profile variable.

To answer the remaining questions concerning the preservation of the line-profile variability of the primary in the spectra after KOREL disentangling, we need to have a closer look at the residual spectra in the rest frame of the primary (secondary), which are provided by the KOREL disentangling: the disentangled line profiles of the primary and secondary, properly shifted in velocity, are subtracted by the program from each individual spectrum.

To visualise the character of the rapid variations of the primary, we show in the left panel of Fig. 5 the residual spectra in the rest frame of the primary for the night series obtained on 20 July 1997 (HJD 2450650). One can see that the lineprofile variations have varying global line asymmetry as well as very complicated structures of subfeatures. A clear pattern of subfeatures is moving from the blue to the red across the residuals. We note that similar moving features are seen in the residual spectra of other hot line-profile variables, perhaps 


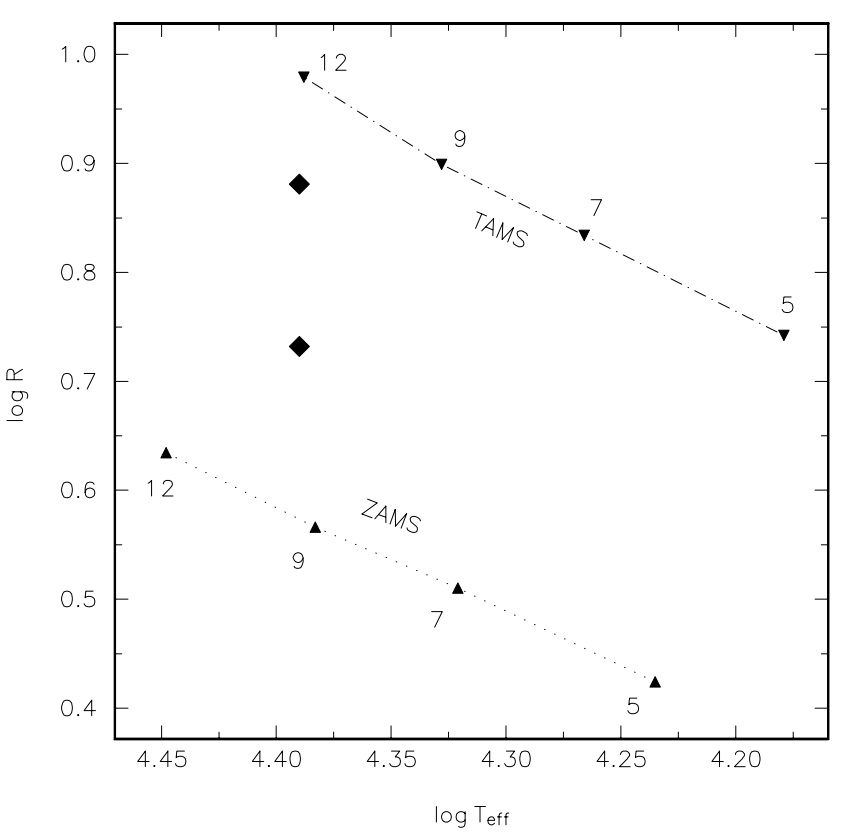

Fig. 6. The maximum and minimum radius of the primary estimated from the Hipparcos parallax are compared with Schaller's et al. (1992) evolutionary models. Masses of the models for the zero-age and terminal-age main sequence are given.

the best example being the archetype line-profile variable $\epsilon$ Persei (cf. De Cat et al. 2000, and references therein).

A Lomb-Scargle period search of the $R V$ (computed as the first velocity moment in the description by Aerts et al. 1992) calculated from the residual spectra in the rest frame of the primary, detects a dominant period close to the 0.19983 period. Moreover, in the prewhitened $R V$ data we also detect the 0.20543 period. The two intrinsic (pulsational) periods reported by Lomb \& Shobbrook $(1972,1975)$ appear clearly in the residual spectra in the rest frame of the primary after KOREL disentangling.

As a comparison, we show in the right panel of Fig. 5 the residual spectra in the rest frame of the secondary for the same series of spectra (HJD 2450650). It is clear that the residuals are much noisier and that no clear pattern of moving features is seen there. No periodicity is found in the $R V$ derived from the residual profiles in the rest frame of the secondary and a search for variations near the 0.19983 period was negative. This shows that we cannot find signs of the intrinsic variability of the primary nor of any other periodicity in the lines of the secondary. This finding corroborates our earlier conclusion that the lineprofile variations are associated with the primary. It also shows that KOREL disentangling is a powerful tool to safely disentangle the individual spectra of binary components even in situations when one of the components is a line-profile variable. We find that the intrinsic variations of the primary do not leave a signature in the disentangled residual profiles of the weak secondary.

Next, we analyzed the intrinsic variability of $\kappa$ Sco in the scalar quantities obtained from the KOREL disentangling.

In Fig. 7 we present periodograms of three scalar quantities ( $R V$, photometry and line intensities). One can see that

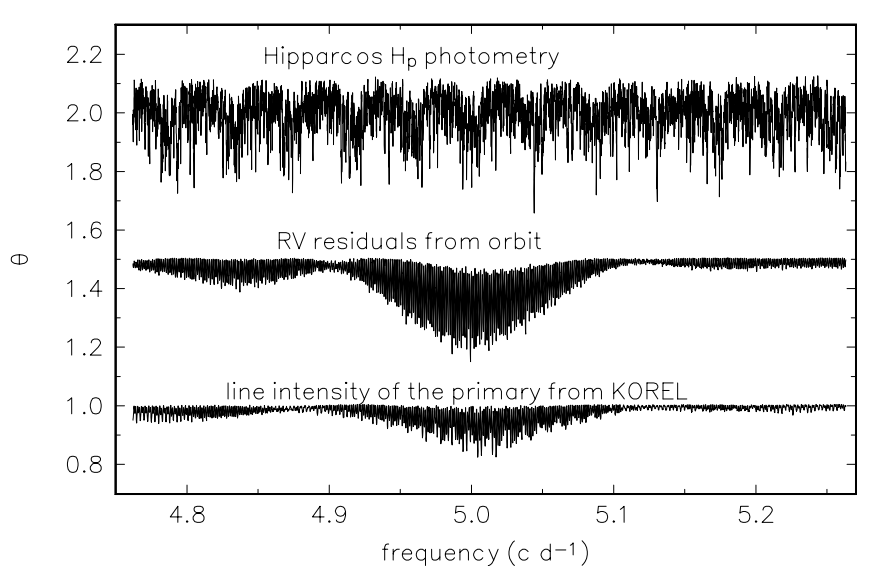

Fig. 7. Stellingwerf's (1978) $\theta$ statistics for the Hipparcos $H_{\mathrm{p}}$ photometry, the $R V$ residuals from the orbit of the primary and for the line intensities of the primary obtained from the KOREL solution, prewhitened for their secular change. One can see the pronounced differences between these periodograms.

all three suffer from strong aliasing problems. Yet, one can note differences between individual periodograms. The periodogram of the KOREL-recovered "light" variations in the bottom panel indicates the presence of a frequency close to $5.004 \mathrm{c} \mathrm{d}^{-1}$, which corresponds to the known 0.19983 photometric period. In contrast, the deepest peak in the periodogram of the $R V$ residuals from the orbit of the primary (affected by line-profile variations) shows a deepest minimum at frequency of $4.9992 \mathrm{c} \mathrm{d}^{-1}$, i.e. for a period of 0.20003 , significantly different from the photometric period. No clear conclusion can be drawn from the periodogram of Hipparcos photometry although we expect that the photometric period should be present in this data set. We therefore show in Fig. 8 the KOREL line photometry converted to magnitudes and the Hipparcos photometry, plotted for the ephemeris based on a sinusoidal fit to the KOREL line photometry. One can see a sinusoidal variation of line intensity variation which has a minimum at the same phase as the much less numerous Hipparcos photometry. The latter is remarkable in the following: the light curve has two clearly defined boundaries: a constant maximum level and an envelope at the bottom, corresponding to light decreases with the largest amplitude ${ }^{4}$. It is known that the amplitude of the light curve for the 0.19983 period varies with a beat period of 7.33 . This means that a simple folding of photometric data obtained over an interval of several years cannot give a clean light curve. However, for oscillations with one frequency and variable amplitude one would expect the observations to be distributed more or less symmetrically around the mean light level. The impression from the Hipparcos light curve instead is that one observes occassional light decreases of a different amount from a constant maximum level, i.e. something seems to shield the stellar radiation during the main oscillation cycle. We admit, however, that this statement is based on very limited Hipparcos data.

\footnotetext{
${ }^{4}$ Note that line intensity variation from KOREL can reflect continuum variations but cannot be directly considered to be identical to a standard light curve - see Hadrava (1997) for details.
} 

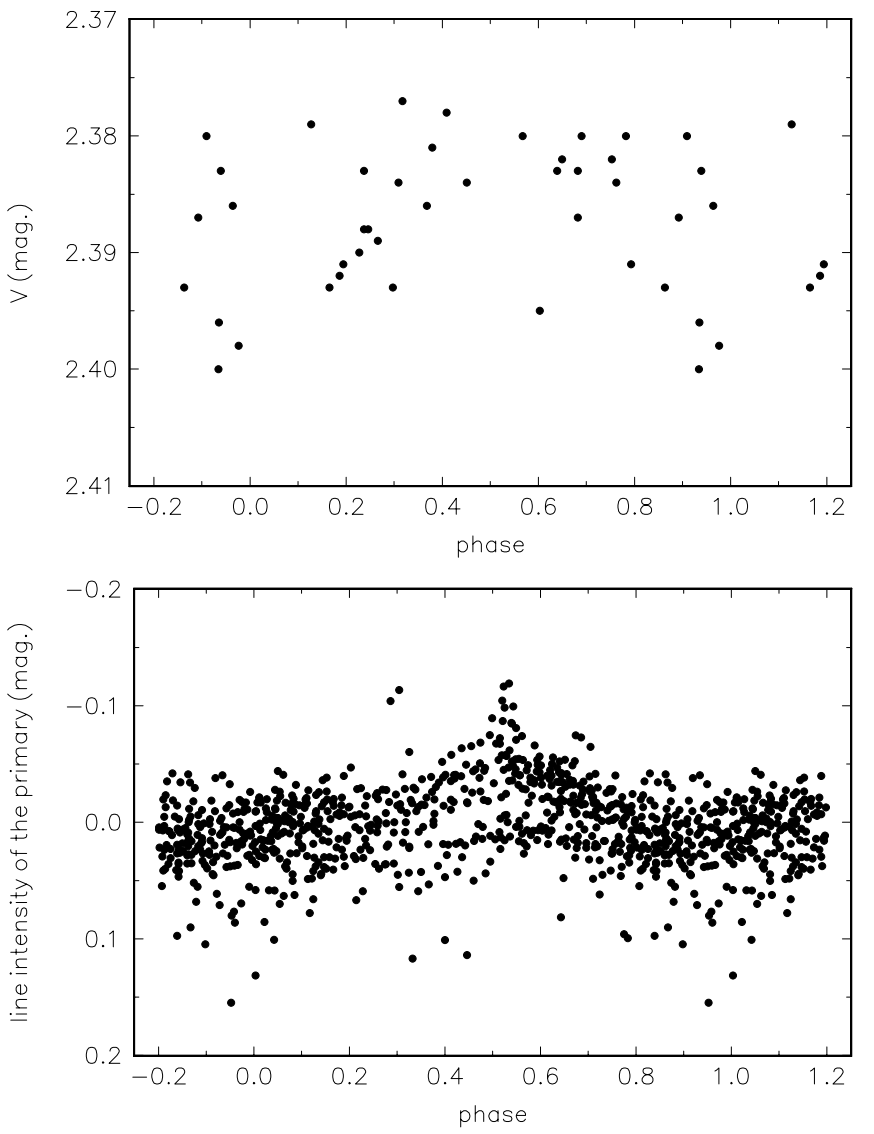

Fig. 8. The Hipparcos $H_{\mathrm{p}}$ photometry, transformed to $V$ magnitude (upper panel) and line intensity variation of the primary from KOREL expressed in magnitudes (bottom panel), plotted vs. phase for the ephemeris of the sinusoidal fit to line intensity: $T_{\text {min.light }}=$ HJD $2449984.1332(41)+0.1998277(15) \times E$.

We also carried out a period search in the line-photometry residuals after their prewhitening for the 0.199828 period. The next best period between 0.15 and 0.21 is a period of 0.1756326 , clearly identical to one of the periods detected in the WIRE photometry.

A detailed analysis and interpretation of the pixel-by-pixel variations in the residual disentangled profiles of the primary and identification of the oscillation modes is beyond the scope of the current paper and will be presented in a follow-up study (Uytterhoeven et al., in preparation).

The result presented here is probably the first application of the method of "line photometry" (Hadrava 1997) to the detection of light variations with a period different from the respective orbital period from spectroscopy without absolute flux calibration.

Figure 9 shows a phase plot of the velocity residuals from the primary orbit vs. phase of the sinusoidal line fit for the best detected period near 0 d20003. Within the accuracy of their determination, the best fit periods from KOREL line photometry and from velocity residuals fulfil exactly the following relation:

$0.20003022^{-1}+195^{\mathrm{d}} \cdot 4579^{-1}=0^{\mathrm{d}} .1998277^{-1}$.

In other words, we find that while the pulsational light variations are detected with their sidereal period, the residual

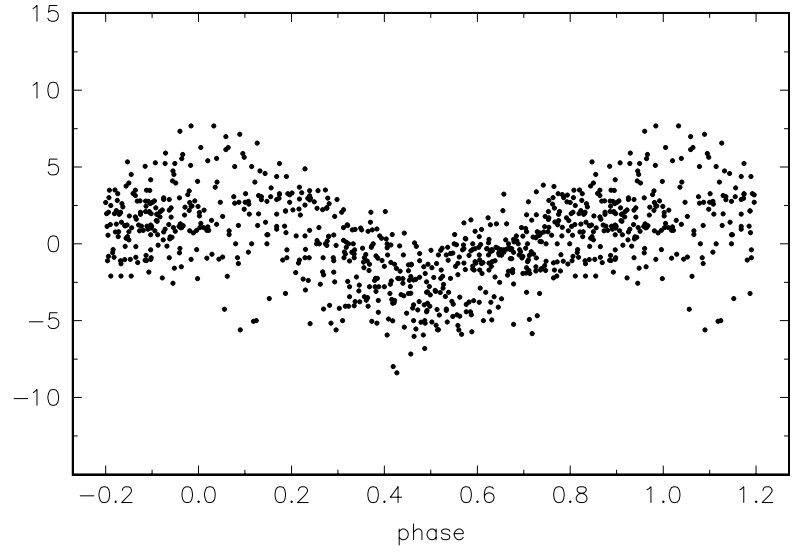

Fig. 9. The $R V$ O-C residuals from the KOREL solution plotted vs. phase for the ephemeris of the sinusoidal fit to them: $T_{\max R V}=$ HJD $2449984.0982(23)+0.20003022(84) \times E$.

$R V$ variations follow the synodic period of the pulsations. Such a possibility was actually predicted for rapid variables in binaries by Harmanec (1999). This deserves further attention and should be taken into account in all subsequent period analyses.

\section{Conclusions}

Using archival $R V \mathrm{~s}$ and especially the modern disentangling technique we substantially improved the knowledge of the basic physical properties of the binary $\beta$ Cep star $\kappa$ Sco. In particular, we derived a new accurate linear ephemeris and a rather tight estimate of the component masses: $10.7-11.9 M_{\odot}$ for the primary and 9.6-10.7 $M_{\odot}$ for the secondary. The comparison of the disentangled line profiles with the synthetic spectra and the assumption that the secondary is a main-sequence star contributing about 10 per cent of light in the visual region leads to an estimate of the rotational period of the primary between 3.56 and 3.68 and rules out spin-orbit synchronization at periastron.

Another important conclusion from the KOREL disentangling is that the observed line-profile variability of $\kappa$ Sco is entirely due to physical variability of the primary star. We shall devote a follow-up study (Uytterhoeven et al., in preparation) to the detailed interpretation of the pixel-to-pixel line-profile variability in terms of oscillations and/or rotational modulation aiming at a unique identification of the observed modes of oscillations. We have already shown here that the scalar quantities derived by KOREL, the $R V$ residuals of the primary from the orbit and the "line intensity", measuring continuum variations, clearly vary with the main known oscillation period of the star and also show some hints of a few other known short periods.

Spectral disentangling is now a well-recognised modern and powerful technique applied to binary-star spectral observations (see Hilditch et al. 2004 for a recent overview of developments in this area). Here, we have shown that KOREL, one of the most elaborate disentangling programs currently available, is remarkably well suited to analyse complex line-profile variations in spectroscopic binaries. Indeed, applying KOREL to a rich set of high dispersion and high- $S / N$ spectroscopic observations of a complicated line-profile variable $\beta$ Cep star $\kappa$ Sco, 
we found that KOREL was able to recover rapid line-profile variations of the primary in the residual spectra derived in the rest frame of this component. This provides an excellent opportunity to carry out a time series analysis of the residual spectra prewhitened for the orbital variations in an objective way. Moreover, all available spectra, not only long night series of observations, become usable for such analyses. The fact that KOREL succeeds in such a situation is by no means obvious since line-profile variability violates one of the principles on which this algorithm is built.

We deliberately tested KOREL on $\kappa$ Sco because of its very complex and so far poorly understood line-profile variations (Uytterhoeven et al. 2001). For this star, the orbital and the intrinsic periods differ mutually for three orders of magnitude. Moreover, the $R V$ amplitudes of the intrinsic variations are for more than one order of magnitude smaller than the orbital one. Such a situation occurs for several other pulsating stars in spectroscopic binaries but certainly not for all of them. It remains to be seen whether KOREL can do an equally good job for systems for which the intrinsic variability occurs on a time scale comparable to the orbital period and/or with an $R V$ amplitude comparable to orbital $R V$ changes. Conceivably, a still more stringent test would be the case of line-profile variations characterised by large profile asymmetries, such as occur in slow rotators, rather than by moving subfeatures. A first attempt to apply disentangling in such a case was carried out by De Cat et al. (2004) for the double-lined slowly pulsating B star HD 123515.

Acknowledgements. We acknowledge the use of the recent versions of the programs FOTEL and KOREL written by Dr. P. Hadrava. This study was initiated thanks to the senior fellowship awarded to P. Harmanec by the Research Council of the Catholic University of Leuven which allowed his three-month stay at the Institute of Astronomy in the Department of Physics and Astronomy. His research was also supported from the research plans J13/98: 113200004 of Ministry of Education, Youth and Sports and AV 0Z1 003909 and project K2043105 of the Academy of Sciences of the Czech Republic. K. Uytterhoeven is supported by the Fund for Scientific Research Flanders (FWO) under project G.0178.02. Finally, we wish to thank the referee, Dr. M. Friedjung, for a careful reading and for suggestions which helped to improve the clarity of the presentation.

\section{References}

Aerts, C., De Pauw, M., \& Waelkens, C. 1992, A\&A, 266, 294

Buscombe, W., \& Morris, P. M. 1960, MNRAS, 121, 263

Campbell, W. W., \& Moore, J. H. 1928, Publ. Lick Obs., 16, 1
Code, A. D., Davis, J., Bless, R. C., \& Hanbury Brown, R. 1976, ApJ, 203, 417

Cuypers, J., Buzasi, D., \& Uytterhoeven, K. 2004, in Variable Stars in the Local Group, ed. D. W. Kurtz, \& K. Pollard, ASP Conf. Ser., in press

De Cat, P., Telting, J. H., Aerts, C., \& Mathias, P. 2000, A\&A, 359, 539

De Cat, P., De Ridder, J., Hensberge, H., \& Ilijic, S. 2004, in Spectroscopically and Spatially Resolving the Components of Close Binary Stars, ed. Hilditch et al., ASP Conf. Ser., in press

De Mey, K. 1997, Ph.D. Thesis, Katholieke Universiteit Leuven, Belgium

Golay, M. 1974, Introduction to astronomical photometry (Dordrecht: Reidel), Astronomical and Space Science Library, 41, 79

Gundlach, J. H., \& Markowicz, S. M. 2000, Phys. Rev. Lett., 85, 2869

Hadrava, P. 1990, Contr. Astron. Obs. Skalnaté Pleso, 20, 23

Hadrava, P. 1995, A\&AS, 114, 393

Hadrava, P. 1997, A\&AS, 122, 581

Harmanec, P. 1988, Bull. Astron. Inst. Czechosl., 39, 329

Harmanec, P. 1998, A\&A, 335, 173

Harmanec, P. 1999, A\&A, 341, 867

Harmanec, P. 2000, in The Be Phenomenon in Early-Type Stars, ed. M. Smith, H. F. Henrichs, \& J. Fabregat, IAU Coll. 175, ASP Conf. Ser., 214, 13

Harmanec, P., Hadrava, P., Yang, S., et al. 1997, A\&A, 319, 867

Heasley, J. N., Wolff, S. C., \& Timothy, J. G. 1982, ApJ, 262, 663

Hilditch, R., Hensberge, H., \& Pavlovski, K. 2004, Spectroscopically and Spatially Resolving the Components of Close Binary Stars, ASP Conf. Ser., in press

Horn, J., Kubát, J., Harmanec, P., et al. 1996, A\&A, 309, 521

Johnson, H. L., Mitchell, R. I., Iriarte, B., \& Wiśniewski, W. Z. 1966, Com. Lunar Planet. Obs., 4, 99

Lomb, N. R., \& Shobbrook, R. 1975, MNRAS, 173, 709

Moore, J. H., Wilson, R. E., \& Paddock, G. F. 1916, Lick Obs. Bull., 287, 37

Perryman, M. A. C., Høg, E., Kovalevsky, J., Lindegren, L., \& Turon, C. 1997, ESA SP-1200, The Hipparcos and Tycho Catalogues

Plaskett, J. S., \& Pearce, J. A. 1931, Publ. Dom. Astrophys. Obs., 5, 1

Popper, D. M. 1980, ARA\&A, 18, 115

Scargle, J. D. 1982, ApJ, 263, 835

Schaller, G., Schaerer, D., Meynet, G., \& Maeder, A. 1992, A\&AS, 96, 269

Shobbrook, R., \& Lomb, N. R. 1975, MNRAS, 156, 181

Škoda, P. 1996, in Astronomical Data Analysis Software and Systems V, ed. G. H. Jacoby, \& J. Barnes, ASP Conf. Ser., 101, 187

Stellingwerf, R. F. 1978, ApJ, 224, 953

Uytterhoeven, K., Aerts, C., De Cat, P., et al. 2001, A\&A, 371, 1035 (Paper A) 